\title{
Giant spin-dependent thermoelectric effect in magnetic tunnel junctions
}

\author{
Weiwei Lin ${ }^{1} \uparrow$, Michel Hehn ${ }^{1}$, Laurent Chaput ${ }^{1}$, Béatrice Negulescu ${ }^{1} \uparrow \uparrow$, Stéphane Andrieu ${ }^{1}$ \\ François Montaigne ${ }^{1} \&$ Stéphane Mangin ${ }^{1}$
}

Thermoelectric effects in magnetic nanostructures and the so-called spin caloritronics are attracting much interest. Indeed it provides a new way to control and manipulate spin currents, which are key elements of spin-based electronics. Here we report on a giant magnetothermoelectric effect in a magnetic tunnel junction. The thermovoltage in this geometry can reach $1 \mathrm{mV}$. Moreover a magnetothermovoltage effect could be measured with ratio similar to the tunnel magnetoresistance ratio. The Seebeck coefficient can then be tuned by changing the relative magnetization orientation of the two magnetic layers in the tunnel junction. Therefore, our experiments extend the range of spintronic devices application to thermoelectricity and provide a crucial piece of information for understanding the physics of thermal spin transport.

\footnotetext{
1 Institut Jean Lamour, Nancy-Université, Boulevard des Aiguillettes, BP 239, Vandoeuvre-lès-Nancy 54506, France. †Present addresses: Institut d'Electronique Fondamentale, Université Paris-Sud, Orsay 91405, France (W.L.); Laboratoire d'Electrodynamique des Matériaux Avancés, CNRS-CEA, Tours 37200, France (B.N.). Correspondence and requests for materials should be addressed to S.M. (email: stephane.mangin@ijl.nancy-universite.fr) orW.L. (email: weiwei.lin@u-psud.fr).
} 
T hermoelectricity has been known since the discovery by T.J. Seebeck in 1821. On one hand, the relation between the thermal and the electrical transport is an essential topic for both fundamental physics and for the future of energysaving technologies ${ }^{1-6}$. On the other hand, the discovery of the giant magnetoresistance effect and the tunnel magnetoresistance effect (TMR) enhanced the interest of the community for spindependent conductivity and gave rise to spintronics and multiple applications $s^{7-9}$. Its interplay with thermal conductivity was introduced to describe the conventional Seebeck effect in ferromagnetic metals ${ }^{10-25}$. The magnetothermoelectric effect has then be studied in magnetic systems such as magnetic multilayers and spin valves ${ }^{10-14}$. Moreover, the thermoelectric effect has also been observed in non-magnetic tunnelling devices such as superconductor-insulator-normal metal (or superconductor) tunnel junctions ${ }^{26,27}$. Recently, thermal spin tunnelling effect from ferromagnet to silicon has been reported ${ }^{28}$. In regard to magnetic tunnel junction (MTJ), there were theoretical works ${ }^{29-31}$ showing magnetothermopower, and Walter et al. ${ }^{32}$ reported first the measurements of Seebeck effect in $\mathrm{MgO}$ MTJs. Their experiments show that the magnitude and sign of the magneto-Seebeck ratio can be changed by laser power modulation ${ }^{32}$.

In this article, we present an experimental discovery of a giant thermoelectric effect in $\mathrm{Al}_{2} \mathrm{O}_{3}$ MTJs. The observed $\mathrm{mV}$ thermovoltage has promising application for the novel magnetic thermoelectric devices.

\section{Results}

Experiment set-up. The studied MTJ consists of a bottom reference layer $\mathrm{Ta}(5 \mathrm{~nm}) / \mathrm{PtMn}(25 \mathrm{~nm}) / \mathrm{Co}_{90} \mathrm{Fe}_{10}(2 \mathrm{~nm}) / \mathrm{Ru}(0.8 \mathrm{~nm}) / \mathrm{Co}_{90} \mathrm{Fe}_{10}$ $(3 \mathrm{~nm})$ and a free layer $\mathrm{Co}_{90} \mathrm{Fe}_{10}(2 \mathrm{~nm}) / \mathrm{Ni}_{80} \mathrm{Fe}_{20}(5 \mathrm{~nm}) / \mathrm{Ru}(4.8 \mathrm{~nm}) /$ $\mathrm{Au}(10 \mathrm{~nm})$ separated by a $2-\mathrm{nm}$ thick amorphous $\mathrm{Al}_{2} \mathrm{O}_{3}$ barrier, as shown in Fig. 1a. To generate a temperature difference between the reference layer and the free layer, one electrode lead was heated using the laser beam from a laser diode with a wavelength of $780 \mathrm{~nm}$ and a tunable power from 0 to $125 \mathrm{~mW}$. The temperature difference between both sides of the $\mathrm{Al}_{2} \mathrm{O}_{3}$ barrier is defined as $\Delta T$, whereas the voltage difference is $\Delta V$. In the linear response approximation, the total electric current $I$ in the presence of $\Delta V$ and $\Delta T$ can be written as ${ }^{10,24}$

$$
I=G_{\mathrm{V}} \Delta V+G_{\mathrm{T}} \Delta T
$$

where $G_{\mathrm{V}}$ is the electrical conductance, and $G_{\mathrm{T}}$ is the thermoelectric coefficient related to the charge current response to the heat flux.

The thermovoltage $\Delta V$ can be measured in an open-circuit geometry, where $I=0$, as shown in Fig. 1b. Considering equation (1) it leads to $\Delta V=-\left(G_{\mathrm{T}} / G_{\mathrm{V}}\right) \Delta T=-S \Delta T$, where $S=G_{\mathrm{T}} / G_{\mathrm{V}}$ is the thermopower (TP) or Seebeck coefficient. $\Delta V$ was measured with a nanovoltmeter at room temperature (RT) with a magnetic field $H$ applied along the in-plane easy axis of the free layer. The thermotunnel current was measured by a sourcemeter connecting the MTJ without any applied voltage, that is, a closed circuit, as shown in Fig. 1c. In the closed-circuit geometry, $\Delta V=0$ and thus from equation (1), $I=G_{\mathrm{T}} \Delta T$. With those two geometries, the influence of magnetization orientations on both spin-dependent electrical conductivity and thermoelectric effect could be studied.

Magnetothermovoltage measurement in MTJ. Figure 2a shows a minor loop of the tunnel resistance $R$ as a function of the in-plane applied field $\mathrm{H}$ for an $\mathrm{Al}_{2} \mathrm{O}_{3}$-based MTJ with a diameter of $80 \mu \mathrm{m}$. The MTJ has a low resistance $R_{\mathrm{P}}=15.9 \mathrm{k} \Omega$ for the parallel $(\mathrm{P})$ magnetizations alignment, and a high resistance $R_{\mathrm{AP}}=22.3 \mathrm{k} \Omega$ for the antiparallel (AP) magnetizations alignment, showing a TMR ratio $\left(R_{\mathrm{AP}}-R_{\mathrm{P}}\right) / R_{\mathrm{P}}=40 \%$.

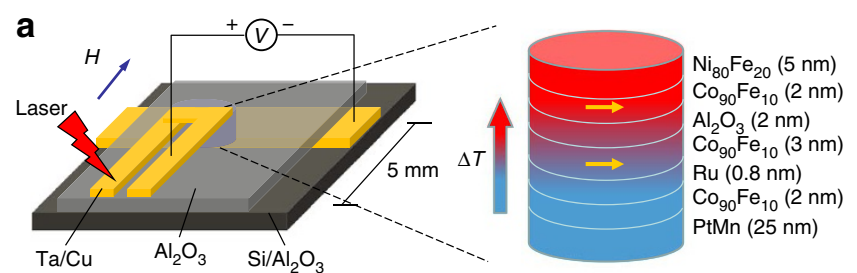

b
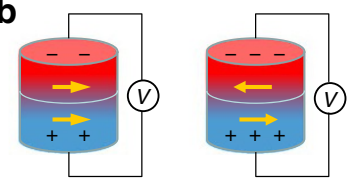

C

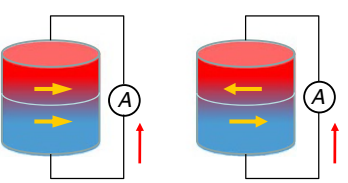

Figure 1 | Schematic of the experiment. (a) The studied MTJ consists of a bottom reference layer $\mathrm{Ta}(5 \mathrm{~nm}) / \mathrm{PtMn}(25 \mathrm{~nm}) / \mathrm{Co}_{90} \mathrm{Fe}_{10}(2 \mathrm{~nm}) /$ $\mathrm{Ru}(0.8 \mathrm{~nm}) / \mathrm{Co}_{90} \mathrm{Fe}_{10}(3 \mathrm{~nm})$ and a free layer $\mathrm{Co}_{90} \mathrm{Fe}_{10}(2 \mathrm{~nm}) / \mathrm{Ni}_{80} \mathrm{Fe}_{20}$ $(5 \mathrm{~nm}) / \mathrm{Ru}(4.8 \mathrm{~nm}) / \mathrm{Au}(10 \mathrm{~nm})$ separated by an $\mathrm{Al}_{2} \mathrm{O}_{3}$ barrier. To generate a temperature difference between both sides of the $\mathrm{Al}_{2} \mathrm{O}_{3}$ barrier, one electrode lead was heated using the laser beam from a laser diode with the wavelength of $780 \mathrm{~nm}$ at a maximum power of $125 \mathrm{~mW}$. The opencircuit voltage was measured by the nanovoltmeter at room temperature (RT) with an applied magnetic field $\mu_{0} H$ up to $0.3 T$ along the in-plane easy axis. (b) In the presence of the temperature difference $\Delta T$ in the MTJ, the generated thermovoltage $\Delta V$ depends on the relative magnetization alignment of the two ferromagnetic layers. (c) The thermotunnel current I was measured by the system sourcemeter connecting the MTJ in a closed circuit without applied voltage.

Then, instead of injecting a current in the MTJ, as sketched in Fig. 1b, the voltage across the MTJ is measured in an open-circuit geometry. The top lead is heated by the laser to generate a temperature difference between the free layer and the reference layer spaced by the $\mathrm{Al}_{2} \mathrm{O}_{3}$ barrier. With the top lead heated, the temperature difference is defined as positive $\Delta T>0$. As shown in Fig. $2 b$, a negative thermovoltage $\Delta V$ is detected in this geometry. While sweeping the in-plane applied field, a sudden $\Delta V$ increase is observed as the free layer magnetization switches and the magnetization configuration changes from $\mathrm{P}$ to AP. In fact, the $\Delta V$ versus $H$ hysteresis loop mimics the $R$ versus $H$ loop. Two thermovoltage levels are clearly defined corresponding to the two magnetization alignments ( $\mathrm{P}$ and $\mathrm{AP})$. The amplitude of the thermovoltage for the AP alignment, $\Delta V_{\mathrm{AP}}$ is found to be larger than that for the $\mathrm{P}, \Delta V_{\mathrm{P}}$. In our case, the $\Delta V_{\mathrm{AP}}$ can reach up to $-1.07 \mathrm{mV}$ while heating the top lead with a $125-\mathrm{mW}$ laser power. The $\Delta V_{\mathrm{AP}}$ is about $310 \mu \mathrm{V}$ as heating the bottom lead with the same laser power. This difference can be understood as different material, thickness and size for the top and bottom leads result in different heat conductivity and dissipation. In the case where the laser heats the bottom lead, that is, $\Delta T<0$, then a positive thermovoltage is measured, as shown in Fig. $2 b$, and an inverse thermovoltage $\Delta V$ hysteresis loop is observed. Note that if the laser is turned off or shines the substrate instead of the leads, the thermovoltage decreases to zero and no influence of the applied field is observed.

For both top and bottom heating, the tunnel magnetothermovoltage ratio defined as $\left(\Delta V_{\mathrm{AP}}-\Delta V_{\mathrm{P}}\right) / \Delta V_{\mathrm{P}}$ is $\sim 40 \%$, which is similar to the TMR ratio. This behaviour suggests that the observed thermoelectric effect mainly results from the thermal spin-dependent tunnelling between both sides of the $\mathrm{Al}_{2} \mathrm{O}_{3}$ barrier. Moreover, the thermovoltage of the lead was measured while heating one end with the maximum laser power, and a value $<2 \mu \mathrm{V}$ was obtained. Thus, the thermovoltage of the lead can be neglected considering the measured thermovoltage in the MTJ.

Figure 3 shows the thermovoltage $\Delta V_{\mathrm{P}}$ and $\Delta V_{\mathrm{AP}}$ as a function of the laser power $P$ in the cases of heating the top (Fig. $3 \mathrm{a}$ ) and 

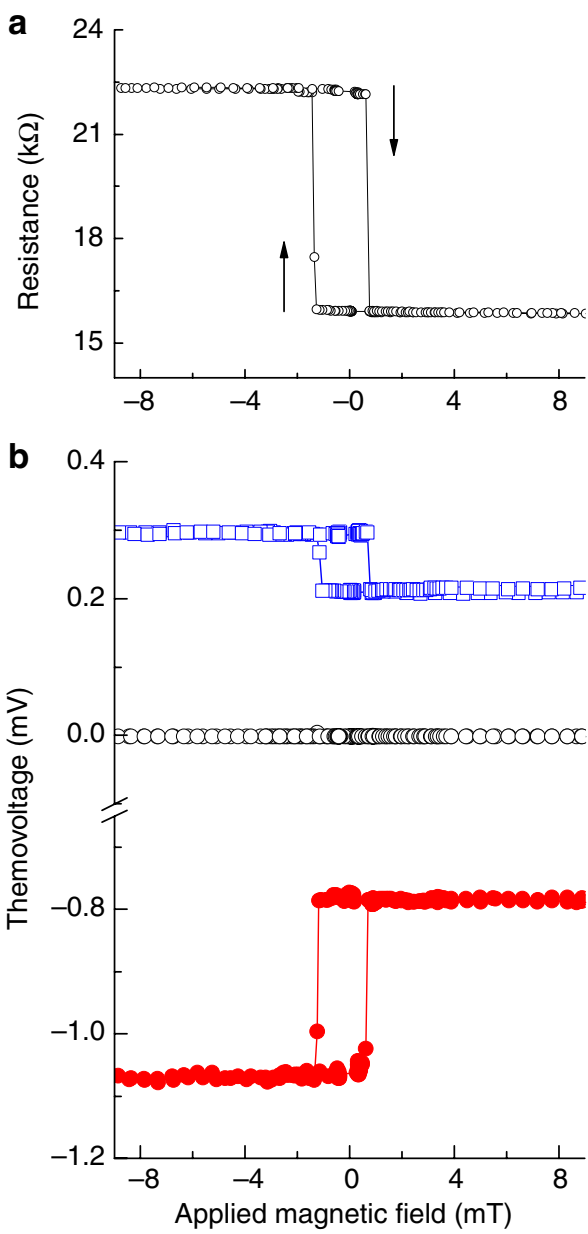

Figure 2 | Magnetic field dependence of the tunnel resistance and the thermovoltage in a MTJ. (a) Minor loop of the tunnel resistance $R$ of an $\mathrm{Al}_{2} \mathrm{O}_{3}$ MTJ with a diameter of $80 \mu \mathrm{m}$ as a function of $\mathrm{H}$ at room temperature, measured with a $0.1-\mu \mathrm{A}$ current. The arrows indicate the sweeping direction of the magnetic field. The MTJ has a low resistance $R_{\mathrm{P}}=15.9 \mathrm{k} \Omega$ for the parallel $(\mathrm{P})$ magnetization alignment, and a high resistance $R_{\mathrm{AP}}=22.3 \mathrm{k} \Omega$ for the antiparallel (AP) alignment. The TMR ratio is $\left(R_{\mathrm{AP}}-R_{\mathrm{P}}\right) / R_{\mathrm{P}}=40 \%$. (b) Thermovoltage versus applied field $(\mathrm{V}-\mathrm{H})$ minor loops. The voltage across the MTJ is measured in an open-circuit geometry with a laser heating the electrodes. As the laser heats the top lead (solid circle), the temperature of the free layer is higher than that of the reference one, that is, $\Delta T>0$, yielding a negative thermovoltage $\Delta V$, whereas a positive $\Delta V$ is observed in the case of the laser heating the bottom lead (open square), that is, $\Delta T<0$. It is noted that the open-circuit voltage is zero in the absence of laser heating (open circle). With sweeping the applied field, $\Delta V$ shows a behaviour similar to $R$. The amplitude of the thermovoltage for the AP alignment, $\Delta V_{A P}$, is larger than that for the $P, \Delta V_{P}$. The $\Delta V_{\text {AP }}$ can reach $-1.07 \mathrm{mV}$ as heating the top lead with a $125 \mathrm{~mW}$ laser power, while the $\Delta V_{A P}$ is about $310 \mu V$ as heating the bottom lead with the same laser power. The tunnel magnetothermovoltage ratio defined as $\left(V_{A P}-V_{P}\right) / V_{P}$ is $\sim 40 \%$, which is similar with the TMR ratio of the MTJ.

the bottom leads (Fig. 3b). One can see that the amplitudes of both $\Delta V_{\mathrm{P}}$ and $\Delta V_{\mathrm{AP}}$ increase with the laser power. The experimental data, obtained for $\Delta V_{\mathrm{P}}$ and $\Delta V_{\mathrm{AP}}$ are not linear but following a $P^{1 / 2}$-like behaviour for the top lead and bottom lead heating. In our understanding this $P^{1 / 2}$ behaviour is not the signature of a microscopic process, but rather is due to the dependence of the temperature difference and of the Seebeck coefficient with $P$. Owing to power dissipation, the temperature difference follows a $P^{\alpha}$ law with $\alpha<1$,
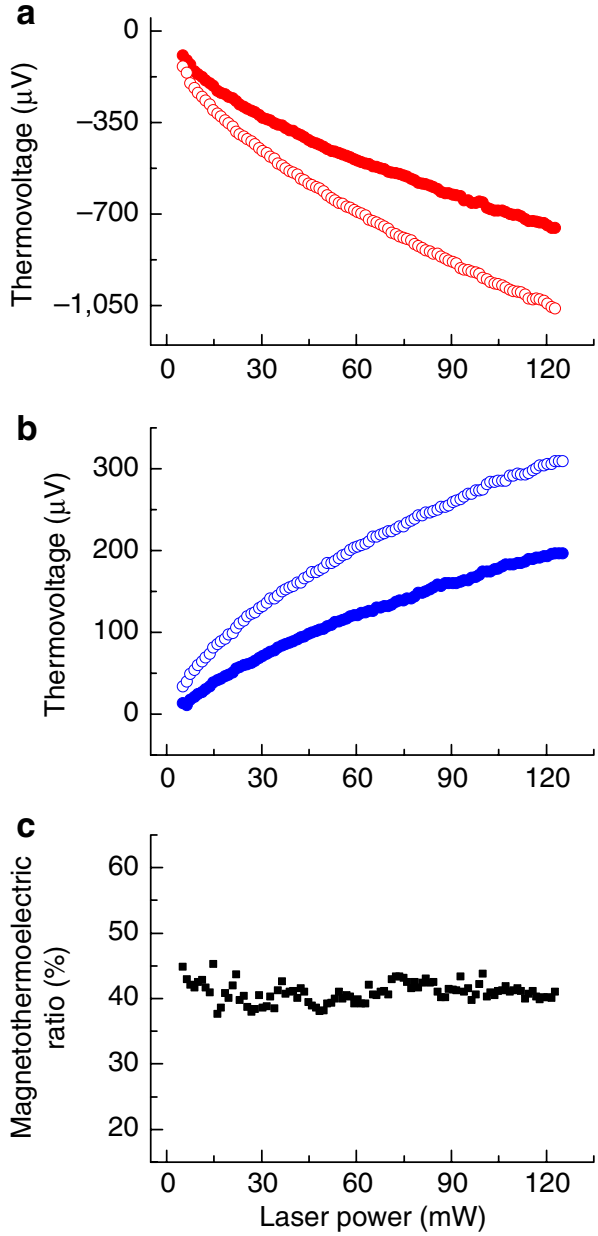

Figure 3 | Laser power dependences of the magnetic thermovoltage and magnetothermoelectric ratio in the MTJ. The thermovoltage $\Delta V_{P}$ (solid circles) and $\Delta V_{A P}$ (open circles) as a function of the laser power $P$ in the cases of heating the top lead (a) and the bottom lead (b). It is found that $\Delta V_{P}$ and $\Delta V_{A P}$ behave like $P^{1 / 2}$. (c) The magnetothermovoltage ratio $\left(\Delta V_{A P}-\Delta V_{P}\right) / \Delta V_{P}$ as a function of the laser power. It is $\sim 40 \%$, which is very close to the TMR ratio, and is constant with the laser power.

and the Seebeck coefficient is itself a complicated function of the temperature, and thus of $P$. The magnetothermovoltage ratio $\left(\Delta V_{\mathrm{AP}}-\Delta V_{\mathrm{P}}\right) / \Delta V_{\mathrm{P}}$ is $40 \%$, which is close to the TMR ratio, and changes little with the laser power, as shown in Fig. $3 \mathrm{c}$.

Thermotunnel current measurement in MTJ. Figure 4a shows the measured thermotunnel current $I$ as a function of $H$ in a closedcircuit geometry, as described above. Without laser heating, the closed-circuit current is around zero. As heating the top lead with a $125-\mathrm{mW}$ laser power, $I$ reaches $43 \mathrm{nA}$, and $I$ is about $-12 \mathrm{nA}$ when the bottom lead is heated by the same laser power. One can see that the thermotunnel current $I$ is independent on the magnetization alignments. However, similarly with the thermovoltage, the laser power dependence of the thermotunnel current also behaves like $P^{1 / 2}$ for both top and bottom leads heating, as shown in Fig. 4b,c. Considering the amplitude, the sign and the magnetic dependence of the measured signal, the possibility of an artefact coming from the known light-induced phenomenon could be ruled out.

\section{Discussion}

From the above experimental results obtained in $\mathrm{Al}_{2} \mathrm{O}_{3}$ MTJs, one can see that the magnetothermovoltage is proportional to TMR, 

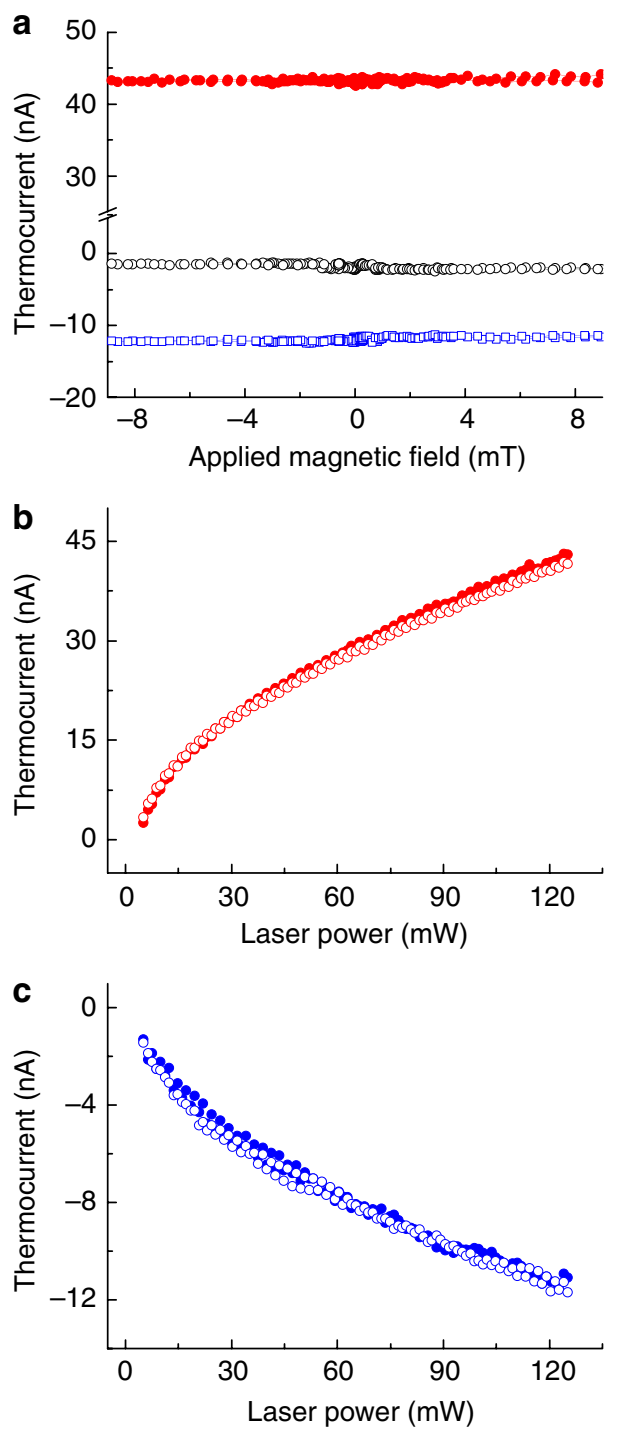

Figure 4 | Magnetic field and laser power dependence of the thermotunnel current in the MTJ. (a) Thermotunnel current versus applied field $(I-H)$ curves in the cases of heating the top lead (solid circles), the bottom lead (open squares) and without heating (open circles). I is independent on the magnetization alignments. $(\mathbf{b}, \mathbf{c})$ The thermotunnel current $I_{P}$ (solid circles) and $I_{A P}$ (open circles) as a function of the laser power $P$ for heating the top and the bottom leads, respectively. It is found that $I_{P}$ and $I_{A P}$ are following a $P^{1 / 2}$-like behaviour.

that is, $\Delta V_{\mathrm{P}} / \Delta V_{\mathrm{AP}}=R_{\mathrm{P}} / R_{\mathrm{AP}}$ whereas the thermotunnel current is independent on magnetizations relative orientations. As the thermovoltage is given by $\Delta V=-\left(G_{\mathrm{T}} / G_{\mathrm{V}}\right) \Delta T=-S \Delta T$, whereas the thermocurrent is given by $I=G_{\mathrm{T}} \Delta T$, assuming that for a fixed laser power $\Delta T$ is constant for the $\mathrm{P}$ and AP configuration, we could define a tunnel TP $S$, which depends strongly on the magnetization alignment of the two magnetic layers. It leads to the conclusion that the coefficient $G_{\mathrm{T}}$ is independent on the magnetization alignments. Consequently, the tunnel magnetothermopower is proportional to TMR in $\mathrm{Al}_{2} \mathrm{O}_{3}$ MTJs, that is, $S_{\mathrm{P}} / S_{\mathrm{AP}}=R_{\mathrm{P}} / R_{\mathrm{AP}}$. It should be said that this behaviour is not conventional, as usually the Seebeck coefficient is not dominated only by the resistance. Indeed, the experiments of other groups ${ }^{32,33}$ show that there is no direct relation between magneto-Seebeck effect and TMR in MgO MTJs, which agree with the ab-initio calculation ${ }^{31}$.

To obtain the value of $S$, the temperature difference between both sides of the tunnel barrier is needed. Unfortunately, it is hard
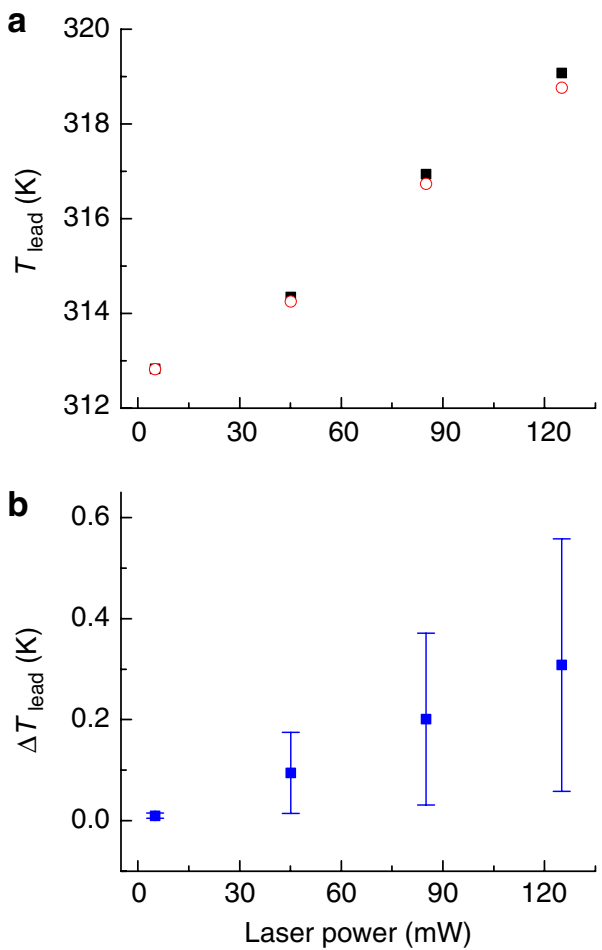

Figure $\mathbf{5}$ | Laser power dependence of the temperature difference of the leads in the MTJ. (a) Temperatures of the bottom lead (solid squares) and the top lead (open circles) as the bottom lead was heated for various laser powers. (b) Laser power dependence of the temperature difference between the bottom and the top leads. The error bars of the temperature difference are given by repeating the measurements.

to directly measure a small temperature difference between a 2-nm barrier. The temperature difference between the bottom and top leads was obtained by measuring the temperature of each lead with a k-type thermocouple connected to a nanovoltmeter. As shown in Fig. 5, for instance with a laser power of $125 \mathrm{~mW}$ heating the bottom lead, the temperatures of the leads are about $319 \mathrm{~K}$, whereas the temperature difference between the top and the bottom leads is $300 \pm 250 \mathrm{mK}$. We could conclude that the temperature difference is smaller than $1 \mathrm{~K}$ between the top and the bottom leads, which could be much smaller between both sides of the tunnel barrier in the MTJ. It should be noted that only part of the heat goes through the MTJ, and because the size of MTJ is much smaller than that of the leads and the 2-nm thickness of $\mathrm{Al}_{2} \mathrm{O}_{3}$ barrier is small comparing with the $60 \mathrm{~nm}$ thickness of the whole multilayers, the vertical temperature difference across the 2-nm $\mathrm{Al}_{2} \mathrm{O}_{3}$ barrier should be smaller than the one measured between the two leads. For a 125-mW laser power, an upper limit for the temperature difference across the barrier can be estimated to be $\sim 100 \mathrm{mK}$. Consequently, from the measured $1-\mathrm{mV}$ thermovoltage, we can estimate that the Seebeck coefficients in $\mathrm{Al}_{2} \mathrm{O}_{3}$ MTJ should be on the order of $1 \mathrm{mVK}^{-1}$ or larger, which is large compared with the conventional metals and semiconductors ${ }^{1}$.

Walter et al. ${ }^{32}$ show the TP in MgO MTJ of $100(1300) \mu \mathrm{VK}^{-1}$ with a $5.3 \mu \mathrm{V}$ measured thermovoltage and a simulated temperature difference across the barrier of $53(4.4) \mathrm{mK}$. Theoretical study by McCann and Fal'co ${ }^{30}$ using inelastic magnon model estimates $55 \mu \mathrm{V} \mathrm{K}^{-1}$, and the ab-initio theory by Czerner et al. ${ }^{31}$ gives values of up to $150 \mu \mathrm{V} \mathrm{K}^{-1}$. This means that giant $\mathrm{TP}$ can be obtained in an $\mathrm{Al}_{2} \mathrm{O}_{3}$ MTJ. 
In the following, we provide a simple model within the linear response theory that agrees with our experimental results. Note that this explanation does not exclude that this behaviour could result from a very peculiar inelastic scattering of the electrons with phonons or magnons, but in the absence of detailed experimental evidence of this type of process we will use a description based on elastic scattering only and find the particularities needed to explain the experimental data. In such a case, it is possible to express the Onsager coefficient $L_{11}=G_{\mathrm{V}}$ and $L_{12}=G_{\mathrm{T}}$ as the moments of order 0 and 1 of the transport function $\sigma(\varepsilon)$,

$$
L_{11}=\int d \varepsilon \sigma(\varepsilon)\left(-\frac{\partial f}{\partial \varepsilon}\right), L_{12}=\frac{1}{(-e) T} \int d \varepsilon \sigma(\varepsilon)(\varepsilon-\mu)\left(-\frac{\partial f}{\partial \varepsilon}\right)
$$

$f$ is the Fermi-Dirac distribution function, and $(-\mathrm{e})$ the electron charge. This approach is well used for bulk thermoelectricity $1,27,34$ and has recently been applied in the context of spin caloritronics in the works by Czerner et al. ${ }^{31}$ and Walter et al. ${ }^{32}$ The function $\sigma(\varepsilon)$ has the physical meaning of an energy-dependent conductivity for the electrons. The quantities $L_{11}$ and $L_{12}$ measure, respectively, the value and the slope of the function $\sigma(\varepsilon), k_{\mathrm{B}} T$ around the Fermi level.

In the case of a $\mathrm{P}$ configuration, $\sigma(\varepsilon)$ is given by

$$
\sigma_{\mathrm{P}}(\varepsilon)=\frac{2 \pi}{\hbar} e^{2}\left\{\left|T_{\uparrow \uparrow}\right|^{2} \rho_{\uparrow}^{L} \rho_{\uparrow}^{R}+\left|T_{\downarrow \downarrow}\right|^{2} \rho_{\downarrow}^{L} \rho_{\downarrow}^{R}\right\}
$$

whereas for an AP configuration,

$$
\sigma_{\mathrm{AP}}(\varepsilon)=\frac{2 \pi}{\hbar} e^{2}\left\{\left|T_{\uparrow \downarrow}\right|^{2} \rho_{\uparrow}^{L} \rho_{\downarrow}^{R}+\left|T_{\downarrow \uparrow}\right|^{2} \rho_{\downarrow}^{L} \rho_{\uparrow}^{R}\right\}
$$

$\rho_{\uparrow}^{L, R}$ and $\rho_{\downarrow}^{L, R}$ are the spin-up and spin-down density of states (DOS) in the left (L) and right (R) leads. $\left|T_{\sigma \sigma^{\prime}}\right|^{2}$ are the tunnelling functions. From $S=G_{\mathrm{T}} / G_{\mathrm{V}}=L_{12} / L_{11}$, it is clear that the TP will be proportional to the resistivity $1 / L_{11}$, if $L_{12}$ is independent of the magnetization orientation, as found in our experiment. In view of equation (2), this requires the slope of the transport function, averaged $k_{\mathrm{B}} T$ around the Fermi level, to be the same in the $\mathrm{P}$ and AP configuration. Notice that this does not preclude for the values of $\sigma_{\mathrm{P}}$ and $\sigma_{\mathrm{AP}}$ to be different and therefore allow observing a TMR.

Unlike for the $\mathrm{MgO}$ MTJs, the Jullière $\operatorname{model}^{7}$ may be appropriate for the $\mathrm{Al}_{2} \mathrm{O}_{3}$ MTJs. Therefore, neglecting the energy dependence of the tunnelling functions, the slopes of $\rho_{\uparrow}^{L} \rho_{\uparrow}^{R}+\rho_{\downarrow}^{L} \rho_{\downarrow}^{R}$ and $\rho_{\uparrow}^{L} \rho_{\downarrow}^{R}+\rho_{\downarrow}^{L} \rho_{\uparrow}^{R}$ should then approximately be the same. Our experimental results would be consistent with DOSs written as $\rho_{\uparrow}=\rho_{0 \uparrow}+\delta \rho$ and $\rho_{\downarrow}=\rho_{0 \downarrow}+\delta \rho$ where $\rho_{0 \uparrow}$ and $\rho_{0 \downarrow}$ are the DOS for an alloy of cobalt with iron, and $\delta \rho$ a spin-independent contribution that can be understood as a resonance. In such a case, with $\rho_{0}^{L, R}=\rho_{0 \uparrow}^{L, R}+\rho_{0 \downarrow}^{L, R}$

$$
\begin{gathered}
\sigma_{\mathrm{P}} \propto \rho_{0 \uparrow}^{L} \rho_{0 \uparrow}^{R}+\rho_{0 \downarrow}^{L} \rho_{0 \downarrow}^{R}+\delta \rho\left(\rho_{0}^{L}+\rho_{0}^{R}\right)+2 \delta \rho^{2} \\
\sigma_{\mathrm{AP}} \propto \rho_{0 \uparrow}^{L} \rho_{0 \downarrow}^{R}+\rho_{0 \downarrow}^{L} \rho_{0 \uparrow}^{R}+\delta \rho\left(\rho_{0}^{L}+\rho_{0}^{R}\right)+2 \delta \rho^{2}
\end{gathered}
$$

Because spin-up and spin-down DOS of bulk cobalt and iron have small slope at the Fermi level on the scale of $k_{\mathrm{B}} T$, it is a good approximation that it is also true for their alloys, if no special atomic order is created, as in our compounds. The energy dependence and the slopes of $\sigma_{\mathrm{P}}$ and $\sigma_{\mathrm{AP}}$ are then dominated by the one of the resonance $\delta \rho$, and therefore is independent on the P or AP configuration. Inserting equations (5) and (6) into equation (2), we obtain the Seebeck coefficients

$$
\begin{gathered}
S_{\mathrm{P}}=\frac{\pi^{2} k_{B}^{2}}{3(-e)} T \frac{\delta \rho^{\prime} / \rho^{L}+\delta \rho^{\prime} / \rho^{R}}{a^{R} a^{L}+\left(1-a^{R}\right)\left(1-a^{L}\right)} \\
S_{\mathrm{AP}}=\frac{\pi^{2} k_{B}^{2}}{3(-e)} T \frac{\delta \rho^{\prime} / \rho^{L}+\delta \rho^{\prime} / \rho^{R}}{\left(1-a^{R}\right) a^{L}+a^{R}\left(1-a^{L}\right)}
\end{gathered}
$$

To obtain these expressions we have used the low temperature expansion of the Fermi function, and the following definitions $\rho^{L, R}=\rho_{\uparrow}^{L, R}+\rho_{\downarrow}^{L, R}, a^{L, R}=\rho_{\uparrow}^{L, R} / \rho^{L, R}$.

The numerator of equations (7) and (8) describes why the thermocurrent of Fig. $4 \mathrm{a}$ is independent of the magnetization alignment, whereas in the denominator we recognize the Jullière expression for the conductance in term of polarizations $a^{L, R}$. This explains the proportionality observed between the magnetoSeebeck effect and TMR in Fig. 2. These formula are also consistent with the large value observed for the thermovoltage, if $\delta \rho$ is a very narrow non-magnetic resonance giving rise to a large $\delta \rho^{\prime}$. This could originate, for example, from non-magnetic impurity states, as they are usually narrow.

In summary, large thermoelectric effect was observed in the MTJ arising from the temperature difference between both sides of a 2-nm $\mathrm{Al}_{2} \mathrm{O}_{3}$ tunnel barrier. The magnetothermovoltage ratio for the $\mathrm{P}$ and $\mathrm{AP}$ magnetization configuration is similar to the TMR ratio in the $\mathrm{Al}_{2} \mathrm{O}_{3}$ MTJ. However, the thermotunnel current is independent on the magnetization alignments. The TP can be estimated to be $>1 \mathrm{mVK}^{-1}$ in the $\mathrm{Al}_{2} \mathrm{O}_{3} \mathrm{MTJ}$, which is larger than that in the metal and semiconductor, suggesting that MTJ can be used as a good thermospin device. The thermospin devices can work in an open-circuit without applying any current or voltage. On one hand, the large change in thermovoltage can be obtained in the presence of a temperature difference through controlling the relative magnetization alignment of the two ferromagnetic layers in the MTJ. On the other hand, the magnetothermovoltage can be used to detect the magnetization configuration even in the open-circuit geometry. The exact mechanism may still be discussed, but we are proposing a description based on elastic scattering to explain qualitatively the experimental results.

This work extends the understanding of the spin-dependent thermal and electrical transport in nanostructures, and has promising potential for the design and application of thermally driven MTJ.

\section{Methods}

MTJ preparation. The MTJ consists of a bottom reference layer $\mathrm{Ta}(5 \mathrm{~nm}) /$ $\mathrm{PtMn}(25 \mathrm{~nm}) / \mathrm{Co}_{90} \mathrm{Fe}_{10}(2 \mathrm{~nm}) / \mathrm{Ru}(0.8 \mathrm{~nm}) / \mathrm{Co}_{90} \mathrm{Fe}_{10}(3 \mathrm{~nm})$ and a free layer $\mathrm{Co}_{90} \mathrm{Fe}_{10}(2 \mathrm{~nm}) / \mathrm{Ni}_{80} \mathrm{Fe}_{20}(5 \mathrm{~nm}) / \mathrm{Ru}(4.8 \mathrm{~nm}) / \mathrm{Au}(10 \mathrm{~nm})$ separated by a $2-\mathrm{nm}$ thick $\mathrm{Al}_{2} \mathrm{O}_{3}$ barrier. The films were deposited on the $400 \mathrm{~nm} \mathrm{Al} \mathrm{O}_{3}$ covered $\mathrm{Si}$ wafers in a DC magnetron sputtering system at RT with a base pressure of $2 \times 10^{-8}$ Torr and a deposition pressures of $2-3$ mTorr. The $\mathrm{Al}_{2} \mathrm{O}_{3}$ barrier was obtained by reactive $\mathrm{rf}$ (radio frequency) oxidation of a 2-nm Al layer at a power of $50 \mathrm{~W}$. The films were annealed for $2 \mathrm{~h}$ at the temperature of $265^{\circ} \mathrm{C}$ and a 1.3 -T magnetic field in a $\mathrm{N}_{2}$ atmosphere oven, and then patterned to circular shape with the diameter varying from 40 to $100 \mu \mathrm{m}$ using the photolithography and ion mill processes. The $200 \mathrm{~nm}$ $\mathrm{Cu}$ and $10 \mathrm{~nm}$ Ta were used as both the bottom and the top leads. The MTJs were measured using a system sourcemeter. The TMR is around $40 \pm 3 \%$ and the resistance-area product is about $22 \pm 6 \mathrm{M} \Omega \mu \mathrm{m}^{2}$ at $\mathrm{RT}$.

Magnetothermovoltage and thermotunnel current measurements. To generate a temperature difference between the reference layer and the free layer, the top lead or the bottom lead was heated using a laser beam from a laser diode with a wavelength of $780 \mathrm{~nm}$ and a maximum power of $125 \mathrm{~mW}$. The laser spot on the lead is around $5 \mathrm{~mm}$ away from the junction. It should be noted that only part of heat pass through the MTJ, because the power is dissipated and the size of the MTJ is much smaller than that of the leads. The thermovoltage was measured by a nanovoltmeter having an internal resistance larger than $10 \mathrm{G} \Omega$ in an open-circuit at RT with an applied magnetic field up to $0.3 \mathrm{~T}$ along the in-plane easy axis. The thermotunne current was measured by a sourcemeter having an internal resistance lower than $100 \mathrm{~m} \Omega$, connecting the $16 \mathrm{k} \Omega \mathrm{MTJ}$ in a closed circuit without applied voltage. 
The thermovoltage of MTJ was also checked by measuring the AC voltage using a lock-in with the same frequency of the $\mathrm{AC}$ laser power. The $\mathrm{AC}$ measurement shows the similar behaviour with the DC measurement.

The temperature difference in between the bottom and top leads was obtained by measuring the temperature of each lead with a k-type thermocouple connected to a nanovoltmeter. To minimize the error, we tried to install the thermocouple as close as possible to the junction. The temperatures of the leads were also checked by measuring the resistance variation of the leads due to laser heating.

The $\mathrm{Al}_{2} \mathrm{O}_{3}$ MTJs with the diameters varying from 40 to $100 \mu \mathrm{m}$ were measured and showed similar behaviours.

\section{References}

1. Mahan, G. Good thermoelectrics. in Solid State Physics (Academic Press, 1997).

2. Cahill, D. G. et al. Nanoscale thermal transport. J. Appl. Phys. 93, 793-818 (2003).

3. Jeffrey Snyder, G. \& Toberer, E. S. Complex thermoelectric materials. Nature Mater. 7, 105-114 (2008).

4. Hochbaum, A. I. et al. Enhanced thermoelectric performance of rough silicon nanowires. Nature 451, 163-167 (2008).

5. Boukai, A. I. et al. Silicon nanowires as efficient thermoelectric materials. Nature 451, 168-171 (2008).

6. Pernstich, K. P., Rössner, B. \& Batlogg, B. Field-effect-modulated Seebeck coefficient in organic semiconductors. Nature Mater. 7, 321-325 (2008).

7. Jullière, M. Tunneling between ferromagnetic films. Phys. Lett. 54A, 225-226 (1975).

8. Chappert, C., Fert, A. \& Nguyen Van Dau, F. Emergence of spin electronics in data storage. Nature Mater. 6, 813-823 (2007).

9. Fert, A. Origin, development \& future of spintronics. Rev. Mod. Phys. 80, $1517-1530$ (2008)

10. Johnson, M. \& Silsbee, R. H. Thermodynamic analysis of interfacial transport \& of the thermomagnetoeletric system. Phys. Rev. B 35, 4959-4972 (1987).

11. Conover, M. J. et al. Magnetothermopower of Fe-Cr superlattices. J. Magn. Magn. Mater. 102, L5-L8 (1991).

12. Piraux, L. et al. Large magnetothermoelectric power in $\mathrm{Co}-\mathrm{Cu}, \mathrm{Fe}-\mathrm{Cu} \&$ Fe-Cr multilayers. J. Magn. Magn. Mater. 110, L247-L253 (1992).

13. Baily, S. A. et al. Magnetothermopower of $\mathrm{Co}-\mathrm{Cu}$ multilayers with gradient perpendicular to planes. J. Appl. Phys. 87, 4855-4857 (2000).

14. Gravier, L. et al. Spin-dependent thermopower in $\mathrm{Co}-\mathrm{Cu}$ multilayer nanowires. J. Magn. Magn. Mater. 271, 153-158 (2004).

15. Scheibner, R. et al. Thermopower of a Kondo spin-correlated quantum dot. Phys. Rev. Lett. 95, 176602 (2005)

16. Serrano-Guisan, S. et al. Enhanced magnetic field sensitivity of spin-dependent transport in cluster-assembled metallic nanostructures. Nature Mater. 5, 730-734 (2006).

17. Fullerton, E. E. \& Mangin, S. Origin of magneto-thermogalvanic V in clusterassembled metallic nanostructures. Nature Mater. 7, 257-258 (2008).

18. Uchida, K. et al. Observation of spin Seebeck effect. Nature 455, 778-781 (2008).

19. Dubi, Y. \& Di Venta, M. Thermospin effects in a quantum dot connected to FM leads. Phys. Rev. B 79, 081302(R) (2009).

20. Hatami, M., Bauer, G. E. W., Zhang, Q. \& Kelly, P. J. Thermoelectric effects in magnetic nanostructures. Phys. Rev. B 79, 174426 (2009).
21. Wang, R. Q., Sheng, L., Shen, R., Wang, B. G. \& Xing, D. Y. Thermoelectric effect in single-molecule-magnet junctions. Phys. Rev. Lett. 105, 057202 (2010)

22. Uchida, K. et al. Spin Seebeck insulator. Nature Mater. 9, 894-897 (2010).

23. Jaworski, C. M. et al. Observation of spin-Seebeck effect in ferromagnetic semiconductor. Nature Mater. 9, 898-903 (2010).

24. Johnson, M. Spin caloritronics \& the thermomagnetoelectric system. Sol. Sta. Comm. 150, 543-547 (2010).

25. Takezoe, Y., Hosono, K., Takeuchi, A. \& Tatara, G. Theory of spin transport induced by a temperature gradient. Phys. Rev. B 82, 094451 (2010).

26. Smith, A. D., Tinkham, M. \& Skocpol, W. J. New thermoelectric effect in tunnel junctions. Phys. Rev. B 22, 4346-4354 (1980).

27. Giazotto, F. et al. Opportunities for mesoscopics in thermometry and refrigeration. Rev. Mod. Phys. 78, 217-274 (2006).

28. Le Breton, J., Sharma, S., Saito, H., Yuasa, S. \& Jansen, R. Thermal spin current from ferromagnet to silicon by Seebeck spin tunneling. Nature $\mathbf{4 7 5}, \mathbf{8 2}-85$ (2011)

29. Wang, Z. C., Su, G. \& Gao, S. Spin-dependent thermal and electrical transport in spin-valve system. Phys. Rev. B 63, 224419 (2001).

30. McCann, E. \& Fal'co, V. I. Giant magnetothermopower of magnon-assisted transport in ferromagnetic tunnel junctions. Phys. Rev. B 66, 134424 (2002).

31. Czerner, M., Bachmann, M. \& Heiliger, C. Spin caloritronics in magnetic tunnel junctions: Ab initio studies. Phys. Rev. B 83, 132405 (2011).

32. Walter, M. et al. Seebeck effect in magnetic tunnel junctions. Nature Mater. 10, 742-746 (2011)

33. Liebing, N. et al. Tunneling magnetothermopower in magnetic tunnel junction nanopillars. Phys. Rev. Lett. 107, 177201 (2011).

34. Chaput, L., Pécheur, P., Tobola, J. \& Scherrer, H. Transport in doped skutterudites: Ab initio electronic structure calculations. Phys. Rev. B 72, 085126 (2005).

\section{Acknowledgements}

We thank French National Research Agency (ANR) for financing the PNANO, ISTRADE and ANR-NSF FRIENDS project, and J.Z. Sun, Eric E. Fullerton and A.D. Kent for the fruitful discussion.

\section{Author contributions}

W.L. conceived and designed the experiments, and performed the measurements and analysis. S.M. supervised the experiment. B.N. prepared the MTJ samples. M.H. and S.A. prepared the additional MTJ films, and F.M. patterned the additional MTJ samples. W.L. and S.M. gave physical explanations and L.C. proposed the theoretical model. M.H. and S.A. contributed to the helpful discussions. W.L., L.C. and S.M. wrote the article

\section{Additional information}

Competing financial interests: The authors declare no competing financial interests.

Reprints and permission information is available online at http://npg.nature.com/ reprintsandpermissions/

How to cite this article: Lin, W. et al. Giant spin-dependent thermoelectric effect in magnetic tunnel junctions. Nat. Commun. 3:744 doi: 10.1038/ncomms1748 (2012). 